\title{
Towards Routinely Using Virtual Reality in Higher Education
}

\author{
Tim A. Majchrzak and Jaziar Radianti \\ University of Agder, \\ Kristiansand, Norway \\ \{timam,jaziar.radianti\}@uia.no
}

\author{
Jennifer Fromm \\ University of Duisburg-Essen, \\ Duisburg, Germany \\ jennifer.fromm@uni-due.de
}

\author{
Michael Gau \\ University of Liechtenstein, \\ Vaduz, Liechtenstein \\ michael.gau@uni.li
}

\begin{abstract}
Virtual reality promises to be a tool that can improve higher education. Immersive virtual environments offer the chance to enrich courses with experiential learning experiences. The technological possibilities evolve rapidly and more and more researchers report on adopting virtual reality for learning - albeit such work often has a more or less experimental character. However, the base of knowledge on using virtual reality in higher education is growing; educators who want to employ virtual reality to amend courses, to extend the curriculum with experiential learning, or who want to offer new content enabled through virtual reality, find increasingly rich advice. With this article, we contribute to this advice by providing insights from three research cases. Although these were experimental, their embedding into a larger project enables us to propose recommendations for educators. The ultimate aim of our work is the routine use of virtual reality in higher education.
\end{abstract}

Keywords: Virtual Reality, VR, Immersive VR technology, Higher Education

\section{Introduction}

Virtual reality (VR) comprises "hardware and software systems that seek to perfect an all-inclusive, sensory illusion of being present in another environment" [1]. Compared to other information systems, VR enables a particularly high level of immersion, presence and interactivity [2]. Due to these characteristics, VR promises to be a tool that can improve higher education [3]. It is particularly useful for experiential learning, which can enhance the learning success of students [4]. Immersive virtual environments can provide rich experiences and thereby add value to courses, enabling students to learn in an individual way.

VR has its roots in the field of head-mounted displays (HMDs) [5]. The development of VR technologies in recent years has been rapid. VR hardware and tools for creating virtual environments have been enhanced profoundly. However, current applications of VR in higher education often have an experimental character [6]. There are few guidelines for educators. If a course should be amended with virtual content, educators often need to start from scratch, need to overcome technological hurdles, need to master the technical background by themselves, and need to factor didactic considerations and pedagogic value with little external advice.

The base of knowledge is growing nonetheless, and several studies have looked into benefits and challenges perceived by educators already. Solomon et al. [7] describe that the following benefits of VR are perceived by lecturers: it facilitates participation, students can learn at their own pace, language barriers can be eliminated, socialization between students can be enabled, and it is useful for students with different learning types. However, they also identified challenges [7]: infrastructure and financial aspects, lack of VR skills, and resistance to change. Fransson et al. [8] studied which pedagogical possibilities teachers name for HMDs. For example, increased opportunities to visualise complex processes, and making teaching and learning more interesting through varied and experience-based work were identified. The discussed challenges align with those from the ones named above [8]: 1. economic weakness and possible problems with the technology, 2 . initial learning barriers (getting used to VR takes time), 3. organization and practical enactment for teaching and learning (e.g. class size, number of HMDs available), 4. curricula, syllabuses and expected learning outcomes, and 5. teachers' competences, professional development, and trust. Finally, similar barriers to widespread adoption of VR were identified by Alfalah et al. [9]: a lack of knowledge, additional course preparation time, and reluctance to integrate new technology into curricula.

Undoubtedly, VR can benefit higher education, regardless of it being used to merely amend courses, to extend the curriculum or provide students with additional learning opportunities with experiential learning, or offer new content enabled through VR. However, the challenges need to be overcome. We believe that many 
of the above-named challenges would be mitigated or even removed by providing better guidance for educators. If we can relieve them of background technological work - pretty much like we expect no educator to be able to build a projector to show slides -, creating virtual worlds could become routine. If we could give advice on the didactic, pedagogic, and learning-theoretic underpinnings, educators could decide more rapidly where to use VR, and how.

We describe in this work three research cases from different study programs based on our project on VR in higher education. Although these are experimental, their embedding in the project combined with prior work on literature [6], learning [4], and the market for VR in higher education [10], allows us to draw conclusions. We offer these in the form of recommendations for educators, who soon hopefully can routinely use VR to offer students a richer learning experience.

This paper makes two main contributions. First, we present research cases of using VR prototypes in higher education. While these do not seek to advance technological superiority, they showcase how VR can be used as a vehicle to create a more motivating study experience. These provide lessons learned from early VR applications in higher education. Second, we provide comprehensive recommendations for educators that keep pedagogic requirements and didactic considerations in mind. We thereby support educators who want to add VR experience to their courses.

The remainder is structured as follows. Section 2 provides an overview of the background work including a review of relevant literature. We present three cases of using VR in higher education in Section 3. Then, we discuss the cases and lead over to recommendations in Section 4 before we draw a conclusion in Section 5 .

\section{Status Quo VR in Higher Education}

The current literature on VR in higher education can be divided into three areas. The first stream of research focuses on the design of VR learning applications and their evaluation in terms of usefulness and usability. The second stream tests VR learning applications in short laboratory experiments, often in comparison with traditional learning methods and evaluates their learning outcomes. Only a few studies can currently be assigned to the third stream, which focuses on student perceptions of long-term VR experiences in real-world courses.

\subsection{Stream 1: Design-Oriented VR Studies}

The first research stream consists of design-oriented studies aimed at identifying innovative VR use cases within higher education and the technical implementation of VR learning applications. These studies mostly involve user testing to gather feedback on the use cases and usability.

A recent systematic literature review provides an overview of design-oriented studies on VR in higher education [6]. The review found that most applications targeted the acquisition of declarative knowledge and procedural knowledge in engineering, computer science, and astronomy. For example, a study reported on the development and user testing of a VR-based training system allowing engineering students to assemble and disassemble an engine [11]. Another study presented a VR simulation enabling students to practice robotics programming [12]. Researchers [4] developed and evaluated innovative use cases for VR in higher education through design thinking workshops and derived design principles for VR-based experiential learning applications. A similar approach was taken to design agents in VR for training games [13]. The authors conducted three design workshops with different groups of experts and derived a set of design insights that inform the design of agent-based VR learning environments from a human-computer interaction perspective.

Furthermore, a recent analysis of VR app stores provides a comprehensive overview of VR learning apps already available on the market [10]. The authors concluded that available apps were mostly designed as short-term learning units that can be used more as a supplement to traditional lectures. Taken together, these studies show that a variety of VR application scenarios in higher education are already known, but existing VR learning applications are not necessarily designed for long-term experiences.

\subsection{Stream 2: Short-Term VR Experiments}

The second stream includes short-term experimental studies that measure learning outcomes using quantitative methods such as knowledge tests. A recently published systematic literature review provides a comprehensive overview of such experimental studies comparing the learning outcomes of VR teaching approaches and other forms of learning methods [14]. Most of the 29 included studies found a positive effect of VR use compared to non-immersive methods. However, the authors also emphasized that most of the studies were short-term experiments focusing on science and engineering subjects. For example, Detyna and Kadiri [15] conducted three trial runs of earth simulations using high-end VR hardware. In a survey, the students reported that the short VR simulations enhanced their understanding of topography and engagement with the topic. Likewise, Pande et al. [16] conducted a quasi-experiment in a 
biology course in which bachelor students viewed a total of three simulations either as video or with a VR headset. The comparative study showed a greater increase in knowledge gain and improved knowledge retention in the VR condition. In another study, researchers designed and developed a game-based immersive VR learning environment to study mathematical topics [17]. They integrated knowledge of quadratic functions into the platform and let seventh graders play the game. The results of their experiment showed significant improvements in mathematical achievement and learning motivation applying the VR game in primary and secondary education.

\subsection{Stream 3: Long-Term VR Experiments in Real-World Courses}

To date, only few studies examined student perceptions of VR in higher education over an extended period of time. We identified recent long-term studies in which VR was integrated into real courses and student perceptions were collected via observations, reflections, short surveys, or interviews [18, 19, 20, 21].

Hodgson et al. [18] reported on the development, implementation, and evaluation of immersive learning experiences using $360^{\circ}$ videos. One video provided an immersive view of a patient consultation process and the second video provided a field trip to a historical site. The authors emphasized the importance of multiple cycles of testing to progressively improve the experience.

In a study of an advanced Chinese language class, students researched information about famous landmarks and presented them in an authentic environment using Google Cardboard and Google Expeditions [19]. Students experienced a variety of benefits, including an increased interest in the course content and an enthusiasm to engage with Chinese culture. However, students also reported challenges in the form of physical discomfort and technical difficulties. The authors recommended that lecturers use an additional router to counteract connection issues and allow participation via smartphone (without Google Cardboard) to reduce physical dizziness.

Another long-term study examined regular use of the HTC Vive over two semesters in a geography class in higher education [20]. During the course sessions, one student visited various locations in Google Earth VR and the view was shared with the rest of the class using a projector. Overall, students were positive about the technology, but some were anxious to try the VR headset in front of the entire class. The lecturer reported logistical problems in setting up the VR hardware in the classroom. The authors, therefore, recommended that lecturers address students' fears and permanently install the VR equipment in a dedicated room.

In a further study, authors reported experiences with a computer science lecture in which students participated from home using VR headsets and Mozilla Hubs [21]. Overall, students tended to prefer in-person classes but rated the VR course significantly better than video conferencing lectures. Students' perceptions of the VR experience were negatively impacted by feelings of motion sickness, and students encountered technical issues such as audio and video glitches. The authors also suggested additional features that could help lecturers such as the implementation of a clock, duplicate view of slides, and a virtual notebook.

\subsection{Intermediate Conclusion}

Our study contributes three novel aspects. First, we add to the limited body of research in which VR is used in real-world courses for an extended period of time. Second, we report on three diverse research cases, allowing us to address general but also context-specific challenges and solution approaches. Third, we focus not only on student experiences but also on our experiences as lecturers. This results in recommendations to help lecturers use VR in higher education teaching.

\section{Cases}

This section compiles three cases of VR application in higher education. All three have an experimental and exploratory nature, and seek to motivate students as the common denominator. A comparison of the cases is given in Table 1.

\subsection{Case 1: VR Flipped Classroom}

In the first case, we intended the use of VR to increase student engagement in online teaching and create a sense of genuine social interaction in times of the COVID-19 pandemic. We used VR in a course called Communication \& Collaboration Systems where students learn about challenges and success factors associated with adopting such technologies. We used the VR meeting app Spatial [22] in conjunction with a flipped classroom format where students prepared learning materials at home and deepened their understanding through group work during the course session. The VR experiment also allowed students to learn through their own experience using innovative communication and collaboration technologies. Nine students participated in nine VR course units over a three-month period. The same course was offered to 28 additional students in the video conferencing tool BigBlueButton [23], allowing for a comparison of experiences. 
Table 1. Comparison of the cases

\begin{tabular}{|c|c|c|c|c|c|c|}
\hline Case & Data Collection & Setting & Applications & Class / subject & Participants & Used HW (price) \\
\hline$\overline{1}$ & $\begin{array}{l}\text { Observation notes, } \\
\text { open-ended survey }\end{array}$ & at home & Spatial (free) & $\begin{array}{l}\text { Bachelor lecture - } \\
\text { Collaboration Systems }\end{array}$ & 9 students & $\begin{array}{l}\text { Oculus Quest (450€ } \\
\text { per piece) }\end{array}$ \\
\hline 2 & $\begin{array}{l}\text { Observation notes, } \\
\text { open-ended survey }\end{array}$ & at home & $\sqrt{R}$ (free) & $\begin{array}{l}\text { Master lecture } \\
\text { Management IS }\end{array}$ & 19 students & $\begin{array}{l}\text { Oculus Quest (450€ } \\
\text { per piece) }\end{array}$ \\
\hline$\overline{3}$ & $\begin{array}{l}\text { Observation notes, } \\
\text { open-ended survey }\end{array}$ & $\begin{array}{l}\text { at home } 1 \\
\text { in class }\end{array}$ & $\begin{array}{l}\text { YouTube 3D (free); } \\
\text { Anne Frank House } \\
\text { (Free); Gadgeteer App } \\
\text { (USD 14.99) }\end{array}$ & $\begin{array}{lr}\text { Bachelor } / & \text { Master } \\
\text { lecture } & \text { IS } \\
\text { Cybersecurity } & \end{array}$ & & $\begin{array}{l}\text { Oculus Quest (450€ } \\
\text { per piece) }\end{array}$ \\
\hline
\end{tabular}

Before the course started, we shipped a standalone VR headset (i.e., Oculus Quest) and a silicone hygiene cover to each student. We including a guide on how to configure the headset and install the app. In Spatial, the lecturer can choose from predefined virtual environments to create several spaces (e.g., auditorium, meeting room). Students and lecturers can upload a selfie to automatically generate a realistic avatar. The Oculus Quest has an integrated microphone allowing students and lecturers to talk to each other. While doing so, the avatars show the actual gestures of the user and automated facial expressions to signal who is speaking. Users can teleport through the virtual environment and switch between different spaces. Lecturers and students can use the web app to upload slides, documents, and pictures into the VR environment. Spatial also offers a wide variety of features that can be used to brainstorm ideas (e.g., whiteboard, sticky notes, scribble, search for images, and 3D objects). The video conferencing tool offered similar features (e.g., audio, webcam, breakout rooms, screensharing, whiteboard).

We devoted the first session entirely to experimenting with the app features. For each following session, we compiled a selection of learning materials such as scientific articles and enterprise blog articles. We provided the students with guiding questions enabling them to focus on specific aspects. Each lesson consisted of three group tasks which were based on Bloom's revised taxonomy [24]. This hierarchical taxonomy distinguishes between different levels of the cognitive domain reaching from recalling information to generating new ideas. Hence, we started each session with a sorting task on the virtual whiteboard. For example, students were provided with statements related to different theories they read about at home and were asked to assign the statements to the respective theory. The second task then addressed a higher cognitive level. For example, the students had to compare different theories or evaluate advantages and disadvantages of different technologies, as can be seen in Figure 1. The lecture ended with a group exercise in which the students generated new ideas such as a strategy for the introduction of new technologies or an improved interface design for workspace awareness.

Overall, student feedback on the lecture was very positive. The students were surprised that so many things were already possible in VR and worked better than expected. We observed that the students did not have any major problems and were eager to help each other. Thus, we did not have to take a technical support role and were able to focus on the moderation of group discussions.

Discussions in the VR course were much more lively compared to those in the video conferencing course. Students immediately started to engage in discussions and enjoyed seeing each other as avatar. In addition, students already met in the virtual room before the lecture started and were talking with a "coffee mug object" in their hands. Students have thus attempted to recreate the casual conversations that usually occur before physical lectures, which we have never observed in our video conference course. However, it became apparent that the documentation of results in VR requires more time. Some participants noted that it took them significantly longer to write sticky notes using the virtual keyboard although this improved over time. Nevertheless, we decided to relieve the students of as much typing as possible by preparing whiteboard templates.

Altogether, the students enjoyed the lecture and were willing to participate more than obligatory. They created their own virtual space called the Chatterbox where they could meet after the lecture. In the session before Christmas, they decorated the room with 3D objects. Even if these activities did not directly result in achieving learning outcomes, they kept the students motivated and fostered regular attendance. As a result, the students' final exam grade point average was better in the VR version of the course (arithmetic mean $=1.68$ ) than in the video conference version (arithmetic mean $=2.25$ ), with 1 being the best and 5 being the worst possible grade.

\subsection{Case 2: VR-Learning-Game}

In the second case, we intended to use VR applications in order to study course content, for example, to repeat course content or to prepare for an exam. We used an interactive digital card game called "VR-Learning-Game", which is an extension of the AltspaceVR [25] platform and open-source available 


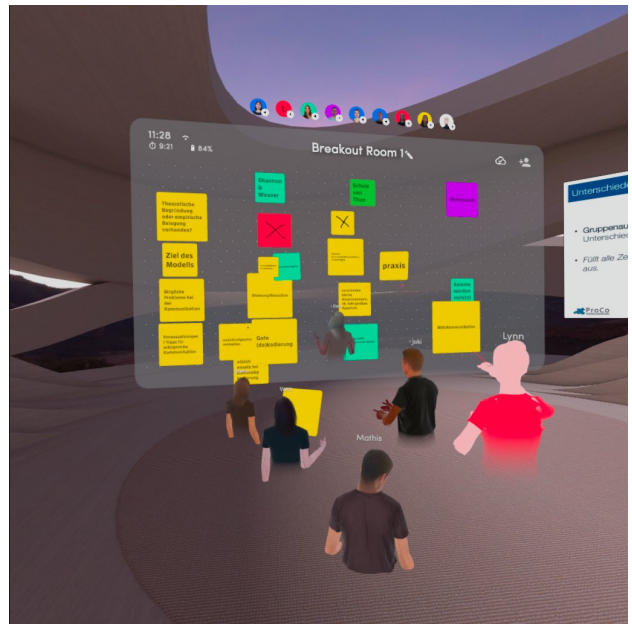

Figure 1. Students compare communication theories using a virtual whiteboard in Spatial

on GitHub ${ }^{1}$. Digital Card (DC) games are widely used in different levels of education to offer a more compelling, personalized, and exciting learning experience [26]. Furthermore, DCs are powerful tools for learning towards school curriculum by game-construction and gameplay [27]. The multi-player game aims to repeat and foster course content by providing collaboration and communication features as, for example, proposed in Boticki et al. [28] or in George et al. [29]. The "VR-Learning-Game" consists of two phases in order to play the game: (1) setting up the card topics and (2) asking for assigned topics.

In the first phase, students should reflect on topics discussed in the course and should come up with topics for the cards. Such topics can be any theory, concept, model, or other important aspects from the related course content. In the second phase, students play the game in VR using the topics derived from the first phase. Every participant gets a virtual avatar and enters a virtual room where the game will be played. Each player can pick a card without knowing the topic. After selecting a card, the topic is shown above the avatars' head, only visible for the other players but not for oneself. Each player needs to find out their topic by asking questions to the group that may only be answered by yes or no. Figure 2 illustrates the game and the assigned topics to players.

Before executing the game in class, we selected a course called Management of Information Systems at our university. Due to the COVID-19 pandemic, the complete course was virtualized. Because of the limitation of having only four VR glasses, not all the students could participate with VR glasses. Some students were using their own VR glasses and others were participating by using their desktop PCs. To facilitate the game

\footnotetext{
${ }^{1}$ https://github.com/donmiguel/VR-Learning-Game
}

installation on the students' devices, a short quick-start guide was provided to the participating students in advance. The four available VR glasses owned by the university were distributed to the students in advance, so they could set the glasses up and get familiar with them.

In the selected course, we used one of the last sessions to play the game as a preparation for the upcoming exam. The participation was voluntary and in total 19 students took part. We started the class by introducing the game to the students and asked them to scan the course manuscripts in order to identify the main concepts, models, constructs, or other learnings of the course. We asked each student to hand in one to three topics that are perceived as important in their opinion. We collected more than 34 topics which we added to the game.

Next, we met in AltspaceVR and did a short introduction to the virtual environment. After all the students got familiar with AltspaceVR, we split up into two groups and played the game. We played various rounds. During the hour of playing, students moved around in the prepared virtual environment and could switch the group or meet in different virtual rooms.

Entering the virtual room, the students quickly began to walk around and explore the environment. They tried to communicate with each other and got familiar with the system very quickly. Starting the game, the interaction began, and the students gathered in a circle. We observed that all students participated and tried to find out the assigned topic. During the game, we observed a high engagement of the students and they used intensively social interaction features like chatting, personal messages, or expressing emotions using smiles or thumb-up icons provided by AltspaceVR.

In the discussion afterward, we observed that the students had fun playing the game and they stated that VR applications could serve as a good alternative to web-conferencing systems especially in the current situation with intense online teaching. However, some difficulties of integrating VR into the daily teaching routine were also mentioned. For example, doubts were raised about the glasses and their comfort, especially when wearing them longer than just an hour. In addition, some students experienced technical issues with slow internet connections and thus gameplay problems.

\subsection{Case 3: Cybersecurity on $360^{\circ}$ Video}

In the third case, we intended to use VR for exploring its use for enhancing teaching and learning of cybersecurity in a course titled Security Management of Information System Development. We used one session of the course curriculum for this experiment, to let the students feel and experience how cybersecurity has been 


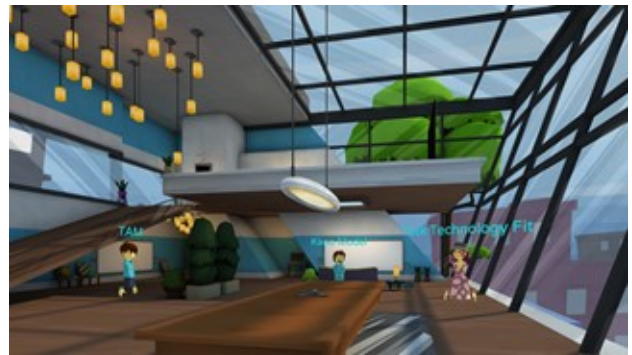

Figure 2. Students playing the VR-Learning-Game to study course content

presented in a virtual world. The VR experiment thereby served a twofold purpose as experiment and learning experience. As a side effect, this provided a realistic setting for the experiment.

The experiment was proposed as a co-design process and tailored with an assignment. The assignment was about providing the design requirements that allow people to learn cybersecurity via VR. The students were required to apply their knowledge and tailor it into the design as one of the course deliverables, working in groups of four students. 12 students participated in the experiments, and 19 students engaged in the assignments. Some students did not participate physically due to health issues. In line with the curriculum of this course, after the experiment we challenged the students to suggest three designs in the form of paper prototypes with adequate explanations such as target group, learning elements, usage descriptions, and the design elements.

We used Oculus Quest headsets during the experiment. As the experiment was conducted in a COVID-19 situation, we carefully applied all measures required during the pandemic time. The measure supports were to use hand sanitizer before touching the VR headset, to clean the headset with disinfectant after each use, to wear a disposable mask for VR headsets, and to use face masks during the experiment. In addition, the experiment was conducted by only two persons at a time with adequate distance between them.

Note that by the time of testing, there were only few apps for learning targeting higher education in the Oculus Quest VR app market [10], and especially for specialized topics such as cybersecurity. Thus, prior to the testing, we conducted preparation works and came up with the idea of watching $360^{\circ}$ videos related to cybersecurity on the headset, which were accessible, but did not go into as much technical depth on the cybersecurity topic. Nevertheless, this option provided all students an opportunity to passively immerse into the VR world and observe, e.g., control room cybersecurity monitoring and response, or critical infrastructure monitoring in a highly realistic environment. But such passive observation posed a risk that the students might underestimate the

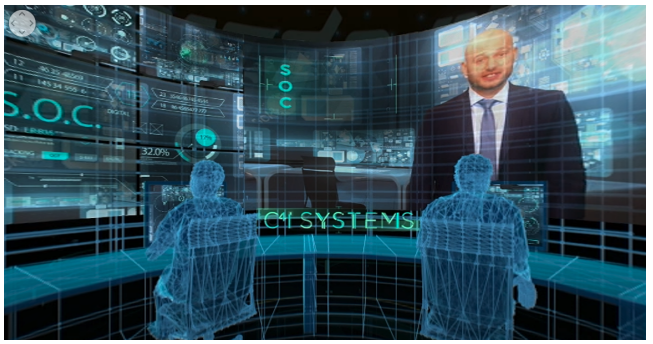

Figure 3. Experiment with $360^{\circ}$ video where the student can virtually observe the situation inside the Security Operation Centre (SOC)

power of VR as a learning tool.

To enhance their VR experience to go beyond passive observation, we also provided alternative learning apps such as 1) the Anne Frank House Museum App that allowed the user to move and walk around from one to another room, to learn about the history of Anne Frank and experience where she used to live, and 2) the Gadgeteer App that is listed as an educative app where the users can experience to hold different tools available in the VR environment, touch, grasp, "feel" the weight of objects, take action, and observe the effects of their choices. The disadvantage was that these two apps were not related to cybersecurity. The purpose was rather to show further what would be possible in the virtual world.

During the experiment in the teaching session we conducted a short survey to map the previous experience dealing with VR headsets. Only a few students had prior experience with HMDs. Some had experience using Google Cardboard. As we anticipated this situation, beginner-to-VR students were recommended to use the YouTube app prepared in the VR headset and find interesting cybersecurity material using voice search. We prepared suggested key words. Some students with more experience preferred to try other apps than the YouTube app or tried to use all possible apps available in the VR headsets (see Figure 3 illustrating the experiment process). In the beginning, the students got an explanation of the overall context and purposes of this experiment. Guidance on how to use the devices (e.g., different buttons on the handhelds) was provided. The students also got an explanation of the different apps available in the VR headsets.

Referring to Bloom's taxonomy on learning objectives [24], the post-experiment assignment was designed to bring the student from lower thinking skills such as remembering and understanding into higher thinking skills such as applying (use information in a new situation), analysing (take apart the known and identify relationships), evaluating (examine the information and make judgments), and creating (use the information to create something new). Thus, although the VR 
experience beyond YouTube $360^{\circ}$ video was not about cybersecurity, the students were encouraged to use and reflect this immersive experience and apply the information in a new context; in this case, to create mock-ups of app ideas for learning about cybersecurity. In total, we received $15 \mathrm{VR}$ mock-ups, including a detailed explanation, goal, and learning points. Based on the quality assessment of the mock-up and the depth of the descriptive explanation and learning goal of the app mock-up, at this point we considered that the majority of the students developed a higher level of critical thinking.

Positively, the students considered that VR could be an alternative to various traditional lecture type courses. VR could be used for telling a story and feeling included in what is happening in a specific situation. Moreover, the app ideas suggested that students considered it positive to step into roles and situations otherwise inaccessible.

Negatively, the students mentioned that VR was quite troublesome, which may cause resistance toward the idea of learning using VR. Some were experiencing nausea and headaches when using the VR equipment. VR use in classrooms at the university was considered not ideal during the COVID-19 pandemic, when students should normally learn from home to avoid contacts. Only a few students have access to VR devices; the cost of VR is still considered too high at this moment. VR equipment will not be bought like a PC and considered indispensable for learning. Some students were sceptical of VR for learning cybersecurity, as they found using a laptop computer to be a sufficient tool for this.

\section{Discussion}

In the following, we first give detailed recommendations for educators. We then sketch the limitations of our work before leading to an outlook.

\subsection{Recommendations}

Based on our observations, we have compiled recommendations for the application of VR in higher education by educators. They target educators directly, for example in their role as lecturers, but also generally, for example as facilitators of curricula. These recommendations have been derived from the cases as sketched in Section 3 and the work with students based on these cases. For each recommendation (save for one, which has not been discussed in any form before), we link to results from the literature.

Teaching Concept: Adapt the teaching concept and learning materials to maximize the learning outcome of VR usage. Although using VR will likely be perceived as motivating by students in any case, merely presenting slides in VR provides little didactic value. VR ought to enable collaborative and experiential learning concepts that amend concepts already in place.

In a recent study, researchers proposed design principles for VR-based experiential learning applications [4]. One design principle also emphasized to utilize the strengths of VR (such as immersion and 3D visualizations) yet to understand what other media can do better (as a simple example: PowerPoint is arguably better to present slides than a VR application).

Testing: Evaluate different VR apps before using them with students. For now, evaluation will need to be carried out with colleagues; with increasing use of VR in teaching, best practices could be compiled and experience with educational apps exchanged.

Yoshimura and Borst [21] identified some missing features of social VR apps that could support teachers (e.g., a missing clock). We can confirm this observation from own experience; some apps lack a clock or an easy-to-use whiteboard where you would have expected one. Such shortcomings cannot be easily noticed (or even mitigated) prior to using an app but will rather reveal themselves when testing it. In alignment with the literature on software testing (cf., e.g. [30]), testing can be seen as an activity that creates value.

Accessibility: Provide standalone VR headsets (such as the Oculus Quest) for home usage and include measures for hygienic usage. This allows students to become familiar with the technology in a private, safe environment. Hygienic use can be supported with covers for parts of the devices that touch skin. If possible, provide VR apps that can also be used without headsets if medical conditions (e.g. epilepsy and wearing a pacemaker) prevent students from using these.

Several authors $[7,8,9]$ have suggested that teachers perceive financial barriers to VR adoption. Scrivner et al. [31] proposed the use of low-cost Google Cardboard, however, they also found that the low resolution can lead to feelings of dizziness. Standalone headsets can be expected to be more accessible in terms of finances (i.e., investment to be made) because they do not require an additional high-quality computer to function. In another perspective on accessibility, Xie et al. [19] reported that some students suffered from physical dizziness and recommended to provide students with an alternative access to the learning content (e.g., use apps that cannot only be viewed with VR headsets but also via desktop computers or mobile phones).

Preparation: Prepare the VR environment carefully. Students should not have to waste time on administrative tasks or struggle with technological difficulties. Besides rigorous testing, providing examples such as whiteboard templates can aid this task.

Previous research $[20,31]$ reported logistical issues 
with installing the VR setup in the given rooms at the university (e.g., missing adapters). Hagge [20] recommended a permanent setup, so that lecturers do not need to spend much time on preparation. However, the preparing inside the VR environment (e.g., whiteboard templates) is equally important, which has rarely been considered in previous studies. This aspect is in line with a prior study with teachers [9], who stated that the increased effort required to prepare VR courses hinders the adoption. Our own observations confirm that this fear is justified! Preparation takes a lot of time and the help of a student assistant was necessary - this extra mile is, however, also worth it due to an increased value of the VR experience. In this sense, testing and preparation go hand in hand.

Tutorial: Provide students with a quick-start guide. While the VR app and its usage on the headset should be as intuitive as possible, setting up the equipment should be accompanied with a short guide. The educator should make sure that the selected $\operatorname{app}(\mathrm{s})$ are properly installed before the first lecture. Since VR is not yet routinely used at most universities, students cannot be expected to successfully set up the equipment on their own (as would be naturally expected for their computers). Nevertheless, it should be mentioned that in our case, students were eager to experiment and supported each other in using the technology. Prepare a short introduction area for students to familiarize with VR, the app(s) in use, and the specific functionality (such as navigating in VR) before starting the actual lecture. This prevents frustration and may especially aid students who would find a virtual encounter with other students intimidating.

Prior works [7, 8, 9] found that teachers perceive the initial learning curve for teachers and students as an adoption barrier. In alignment with this, Hagge [20] recommends explaining the VR hardware to the students in order to reduce anxiety related to the new technology.

Short Experiences: Keep VR units short. Despite progress, VR glasses are not very comfortable to wear for an extended time. Moreover, motion sickness problems need to be prevented (cf. with $[32,33]$ ).

Several studies $[19,20,21]$ confirmed that some students feel physical dizziness, particularly after prolonged use of VR equipment.

Small Groups: Limit the size of groups. It currently is feasible to use VR in seminar-like settings with groups of up to ten students. This avoids distractions and allows solving technical problems. With more routine, scaling up should be possible; for the next few years, scaling to courses where more than a few tens of students use VR at the same time seems unrealistic for economic reasons.

Fransson et al. [8] highlighted various challenges related to the practical enactment of VR teaching relating to the group size. They name, for example, class size, the available number of HMD devices, teachers and support staff, the availability of group rooms, and the opportunities to be flexible with locations, group size, schedules, staff and in-house teaching.

Physical Space: Provide ample space. Applying VR in classrooms, we consider 2 by 1.5 meters to be the minimum space needed for students to try out VR without risking collisions and injury. This space should be free of obstacles, including such that without a VR glass would not pose much of a problem (like a floor cable conduit). More space might be needed depending on the app in use. It should also be considered that students may have less space when using VR at home; seated VR applications may then offer more value than room-scale VR.

Our considerations regarding physical space are closely related to those regarding group size; therefore, they align again with the work of Fransson et al. [8].

Space for Fun: Give students space for activities they enjoy to increase motivation and to encourage regular attendance. For example, the VR environment could provide opportunities to socialise, to engage in seasonal activities (such as jointly decorating a virtual Christmas tree), mini-games, and "easter eggs" to discover.

This aspect does not closely link to the literature. In other words: it has not been discussed in related works. However, with a broader view, one might relate this idea to the concept of gamification [34] - or even in general to psychological works that discuss fun and motivation.

Follow-Up: Take screenshots and videos and share them with the students. Thereby, the activities in VR can be documented. This can also help students to preserve the experience and help them with learning for an exam.

Yoshimura and Borst [21] identified some missing features of social VR apps that could support teachers (e.g., missing virtual notepad). The authors also pointed out the problem but did not propose a solution such as taking screenshots as an easy workaround. We also made the experience that at the beginning students were afraid that they would be unprepared for the exam if they could not take notes themselves. This possibly is related to experience from classical exams, where a lot of knowledge needs to be acquired in order to pass instead of the acquired skills being assessed. In our case, over time students trusted the lecturer to take screenshots for them after the lecture and upload them to the learning management platform. Thereby, important state changes in the virtual world were preserved. Of course students could also take screenshots themselves, but it is better when the lecturer has the opportunity to edit the screenshots (to e.g. correct mistakes) and upload a unified screenshot for the whole class. This way, everybody has equal learning conditions for the exam. 
We can summarize our recommendations as arduously preparing VR usage in education, guiding students, considering pedagogic and didactic approaches taken, and making VR a fun and engaging experience.

\subsection{Limitations}

Many papers appeared that report on VR in higher education. So far, few support educators. We attempt to provide such supports, but some limitations remain.

First, our work is based on three research cases and a surrounding project. We acquired deep insights and gained profound experiences. However, it is unrealistic to assume that we can give definite answers to all current challenges of VR in higher education.

Second, our recommendations have not been quantitatively studied. Admittedly, this is a point for future work; it should be mentioned nonetheless that the effectiveness of our recommendations needs to be evaluated in future studies.

Third, our observations are somewhat subjective, as we did not mention validated measurement instruments to assess student and teacher perceptions. We have taken initial steps into this direction, though. For example, in case 3 we asked the students to express the negative and positive aspects they perceived when using VR, their difficulties, and so on. What is yet missing is a uniform, systematic measurements scheme for VR experiments in higher education courses.

Fourthly, the limitations of the state-of-the-art limit also what we can achieve. For example, the current state of the app market for VR in higher education is not very mature [10]. This again calls for additional work.

These limitations do not impair the value of our work, though. In fact, they call for extended future work.

\subsection{An Outlook}

The proposed recommendations and the limitations make clear two outlooks: First, VR in higher education can be beneficial and educators can find support in implementing virtual environments that provide value to students. Second, many unresolved questions remain.

We expect a rapid evolvement in three fields. First, there is no reason to believe that the development of VR hardware has already reached a plateau. It seems more likely that there will be further advancements with dropping prices due to a widerspread adoption. Second, we expect software environments to soon follow the hardware development. Regarding the use in higher education, easy-to-use frameworks that require neither programming nor 3D modeling proficiency would enable quicker developed and more sophisticated VR environments used in teaching and learning. Moreover, if
VR is adopted widely in higher education, even low level development tools would be valuable since we expect that universities could support the creation of VR teaching and learning environments through staff, much like some universities offer units that support video production and the creation of multimedia content. Third, in the spirit of this work we expect progress in the very considerations of why and how VR can be used in higher education.

The latter question will be targeted from a multitude of perspectives, including didactics, pedagogics, and psychology. We eventually expect that learning theory can be extended. Practically, all the technological work as well as the theory-driven work will need to be joined by more experiments that increasingly aim at reproducibility. In addition to the observations we carried out, long-term VR experiments will be needed, which include a quantitative evaluation of learning outcomes.

VR in higher education is yet in its infancy but it is not a bold assumption to see it mature fast. We do not dare to estimate when it will be used routinely, but we are confident that the benefits it can offer will be leveraged much more often than today within the next five years.

\section{Conclusion}

In this paper, we have presented three cases of using VR in higher education. Based on these three cases, we proposed recommendations for educators who want to use VR in their teaching. These recommendations are Teaching Concept, Testing, Accessibility, Preparation, Tutorial, Short Experiences, Small Groups, Physical Space, Space for Fun, and Follow-Up. We have linked each of these recommendations to the literature.

Revisiting the title of our work, our vision is the routine use of VR in higher education by educators. Undoubtedly, many technological, organizational, and educational steps need to be taken until this will become reality. However, we believe that it is equally realistic for VR to become a tool in education as projectors, presentation software slides, and tablet computers have become. Supporting educators with a set of producible recommendations and, thus, giving them a little less to worry about and a little more to embrace, should prove a first leap into this direction.

\section{Acknowledgments}

This project has been funded with support from the European Commission [Erasmus+ grant number 2018-1-LI01-KA203-000107]. This publication reflects the views only of the authors, and the Commission cannot be held responsible for any use which may be made of the information contained therein. 


\section{References}

[1] F. Biocca and B. Delaney, "Immersive virtual reality technology," Communication in the age of virtual reality, vol. 15 , no. 32, pp. 10-5555, 1995.

[2] J. Mütterlein, "The three pillars of virtual reality? investigating the roles of immersion, presence, and interactivity," in 5st HICSS, 2018.

[3] I. Wohlgenannt, A. Simons, and S. Stieglitz, "Virtual reality," Business \& Information Systems Engineering, vol. 62, no. 5, pp. 455-461, 2020.

[4] J. Fromm, J. Radianti, C. Wehking, S. Stieglitz, T. A. Majchrzak, and J. vom Brocke, "More than experience?-on the unique opportunities of virtual reality to afford a holistic experiential learning cycle," The Internet and Higher Education, vol. 50, p. 100804, 2021

[5] I. Sutherland, "The ultimate display," in Proc. IFIPS Congress 65(2):506-508, CUMINCAD, 1965.

[6] J. Radianti, T. A. Majchrzak, J. Fromm, and I. Wohlgenannt, "A systematic review of immersive virtual reality applications for higher education: Design elements, lessons learned, and research agenda,' Computers \& Education, vol. 147, 2020.

[7] Z. Solomon, N. Ajayi, R. Raghavjee, and P. Ndayizigamiye, "Lecturers' perceptions of virtual reality as a teaching and learning platform," in SACLA, pp. 299-312, Springer, 2018.

[8] G. Fransson, J. Holmberg, and C. Westelius, "The challenges of using head mounted virtual reality in k-12 schools from a teacher perspective," Educ Inf Technol, vol. 25, no. 4, pp. 3383-3404, 2020.

[9] S. F. Alfalah, "Perceptions toward adopting virtual reality as a teaching aid in information technology," Education and Information Technologies, vol. 23, no. 6, pp. 2633-2653, 2018.

[10] J. Radianti, T. A. Majchrzak, J. Fromm, S. Stieglitz, and J. Vom Brocke, "Virtual reality applications for higher educations: A market analysis," in 54th HICSS, 2021.

[11] T. Im, D. An, O.-Y. Kwon, and S.-Y. Kim, "A virtual reality based engine training system-a prototype development \& evaluation," in CSEDU, vol. 2, pp. 262-267, 2017.

[12] V. Román-Ibáñez, F. A. Pujol-López, H. Mora-Mora, M. L. Pertegal-Felices, and A. Jimeno-Morenilla, "A low-cost immersive virtual reality system for teaching robotic manipulators programming," Sustainability, vol. 10, no. 4, p. 1102, 2018.

[13] S. Adinolf, P. Wyeth, R. Brown, and L. R. Simpson, "Near and dear: Designing relatable VR agents for training games," 32nd OzCHI, p. 413-425, 2020.

[14] D. Hamilton, J. McKechnie, E. Edgerton, and C. Wilson, "Immersive virtual reality as a pedagogical tool in education: a systematic literature review of quantitative learning outcomes and experimental design," Journal of Computers in Education, vol. 8, no. 1, pp. 1-32, 2021.

[15] M. Detyna and M. Kadiri, "Virtual reality in the he classroom: feasibility, and the potential to embed in the curriculum," Journal of Geography in Higher Education, vol. 44, no. 3, pp. 474-485, 2020.

[16] P. Pande, A. Thit, A. E. Sørensen, B. Mojsoska, M. E. Moeller, and P. M. Jepsen, "Long-term effectiveness of immersive vr simulations in undergraduate science learning: Lessons from a media-comparison study.," Research in Learning Technology, vol. 29, 2021.
[17] A. Shi, Y. Wang, and N. Ding, "The effect of game-based immersive virtual reality learning environment on learning outcomes: designing an intrinsic integrated educational game for pre-class learning," Interactive Learning Environments, pp. 1-14, 2019.

[18] P. Hodgson, V. W. Lee, J. C. Chan, A. Fong, C. S. Tang, L. Chan, and C. Wong, "Immersive virtual reality (ivr) in higher education: Development and implementation," in Augmented reality and virtual reality, pp. 161-173, Springer, 2019.

[19] Y. Xie, L. Ryder, and Y. Chen, "Using interactive virtual reality tools in an advanced chinese language class: a case study," TechTrends, vol. 63, no. 3, pp. 251-259, 2019.

[20] P. Hagge, "Student perceptions of semester-long in-class virtual reality: Effectively using "google earth vr" in a higher education classroom," JGHE, pp. 1-19, 2020.

[21] A. Yoshimura and C. W. Borst, "Remote instruction in virtual reality: A study of students attending class remotely from home with vr headsets," Mensch und Computer 2020-Workshopband, 2020.

[22] "Spatial: Virtual spaces that bring us together," 2021. https://spatial.io/.

[23] “BigBlueButton," 2021. https://bigbluebutton.org/.

[24] L. W. Anderson and K. D. R, A taxonomy for learning, teaching, and assessing: A revision of Bloom's taxonomy of educational objectives. Longman,, 2001.

[25] "AltspaceVR: The place for events," 2021. https://altvr.com/

[26] Rhonda Epper, Anne Derryberry, and Sean Jackson, "Game-based learning: Developing an institutional strategy," research bulletin, EDUCAUSE, 2012.

[27] M. Kordaki and A. Gousiou, "Digital card games in education: A ten year systematic review," Computers \& Education, vol. 109, 2017.

[28] I. Boticki, L. H. Wong, and C.-K. Looi, "Designing technology for content-independent collaborative mobile learning," IEEE Transactions on Learning Technologies, vol. 6, no. 1, pp. 14-24, 2013.

[29] J. George, E. de Araujo, D. Dorsey, D. S. McCrickard, and G. Wilson, "Multitouch tables for collaborative object-based learning," in Design, User Experience, and Usability. Theory, Methods, Tools and Practice, LNCS, pp. 237-246, Springer, 2011.

[30] T. A. Majchrzak, Improving Software Testing: Technical and Organizational Developments. Heidelberg: Springer Verlag, 2012.

[31] O. Scrivner, J. Madewell, C. Buckley, and N. Perez, "Best practices in the use of augmented and virtual reality technologies for sla: Design, implementation, and feedback," in Teaching language and teaching literature in virtual environments, pp. 55-72, Springer, 2019.

[32] J. Munafo, M. Diedrick, and T. A. Stoffregen, "The virtual reality head-mounted display oculus rift induces motion sickness and is sexist in its effects," Experimental brain research, vol. 235, no. 3, pp. 889-901, 2017.

[33] B. Patrão, S. Pedro, and P. Menezes, "How to deal with motion sickness in virtual reality," in 220 Encontro Português de Computação Gráfica e Interação 2015, The Eurographics Association, 2020.

[34] M. Sailer, J. Hense, J. Mandl, and M. Klevers, "Psychological perspectives on motivation through gamification," Interaction Design and Architecture Journal, no. 19, pp. 28-37, 2014. 